\title{
SAR-SIFT: A SIFT-LIKE ALGORITHM FOR SAR IMAGES
}

\author{
Flora Dellinger, Julie Delon, Yann Gousseau, Julien Michel, Florence Tupin
}

\begin{abstract}
The Scale Invariant Feature Transform (SIFT) algorithm is widely used in computer vision to match features between images or to localize and recognize objets. However, mostly because of speckle noise, it does not perform well on synthetic aperture radar (SAR) images. We present here an improvement of this algorithm for SAR images, named SARSIFT. A new gradient computation, yielding an orientation and a magnitude robust to speckle noise, is first introduced. It is then used to adapt several steps of the SIFT algorithm to SAR images. We study the improvement brought by this new algorithm, compared to existing approaches. We present an application of SAR-SIFT for the registration of SAR images in different configurations, especially with different incidence angles.
\end{abstract}

Index Terms-synthetic aperture radar (SAR), remote sensing, SAR image registration, scale-invariant feature transform (SIFT)

\section{INTRODUCTION}

Last generations of earth observation satellites are providing a large amount of high resolution data, both in optical and Synthetic-aperture radar (SAR) domains, resulting in the multiplication of multi-sensors, multi-resolutions and/or multiangles contexts. To jointly exploit these data for classification, $3 \mathrm{D}$ reconstruction, rapid mapping or change detection, featurebased approaches with some particular invariances may be more suitable than pixel-based ones. This paper studies the interest of feature based descriptors for SAR data in particular.

The Scale Invariant Feature Transform (SIFT) [1] is a very classical algorithm for interest points detection and local features description. Due to its efficiency [2], it is widely used in the field of computer vision to localize and recognize objects between images. Its invariances to scale changes, rotations, translations and partially to illumination changes and affine distorsions make it suitable for different kind of applications such as object retrieval, image indexing, stitching, registration or video tracking.

The SIFT algorithm is an interesting option for remote sensing images due to its performances and invariances. The algorithm has been applied mostly to optical images since they have characteristics similar to natural images. Several registration methods [3], [4], [5] use SIFT keypoints as Control Points (CP) to estimate deformation models. Li [3] takes into account the specificity of remote sensing images and

F. Dellinger, J. Delon, Y. Gousseau and F. Tupin are with the Institut MinesTelecom, Telecom ParisTech, CNRS LTCI, 46, rue Barrault, 75013 Paris, France. e-mail: firstname.lastname@telecom-paristech.fr. J. Michel is with the CNES DCTI/SI/AP, 18, avenue Edouard Belin, 31400 Toulouse, France.

This work was supported by a CNES grant. introduces a new matching criterion with scale and orientation restrictions. A multilevel SIFT matching approach is proposed by Huo [4] to register very high resolution images, with the help of RANdom Sample Consensus (RANSAC). Sedaghat [5] adapts the algorithm to obtain space uniformly distributed keypoints and filters the mismatchs by applying a projective model. The SIFT algorithm has also some assets for remote sensing image retrieval or classification applications. Yang [6] uses bag of words (BoW) representation of SIFT descriptors to perform image retrieval of land-use/land-cover classification in high resolution imagery. Image classification is performed by Risojevic [7] by merging representations of Gabor texture descriptors and SIFT descriptors BoW. Object detection is another application field of the SIFT algorithm. Single buildings are detected on very high resolution optical images by Sirmacek [8] by using SIFT keypoints, multiple subgraph matching and graph cut methods. Tao [9] performs airport detection by considering both clustered SIFT keypoints and region segmentation.

While the SIFT algorithm has proven its efficiency for various kinds of applications in optical remote sensing, the situation is different for SAR images. SAR is an active system and has the advantage of acquiring images independently of weather conditions and solar illumination. SAR images are frequently used in disaster situations since they are often the fastest available ones. However images are corrupted by a strong multiplicative noise, called speckle, and data processing is thus made difficult. The SIFT algorithm does not perform well on this type of images. Several improvements have been proposed to improve the algorithm. Some suggest to pre-filter [10] or denoise [11] the images to reduce the influence of speckle noise. Others remove some invariances [12], [13] or modify some steps of the algorithm [14], [15] to improve the performances. Spatial relationships between keypoints are considered by Lv [16] and Fan [17] to suppress false correspondences. To limit the search space, Wessel [18] uses DEM and orbit information, and Xiaoping [19] performs a manual pre-registration. Details and limitations of these algorithms will be developed later in Section II-C. However performances of these newly developped algorithms are still relatively limited and the number of correct matches is not sufficient enough to consider other applications than registration. Most of them do not consider statistical specificities of speckle noise. Considering the field of applications offered by the SIFT algorithm in optical images, it would be of great interest to have a performant SIFT-like algorithm adapted to SAR images. 
We propose in this paper to adapt the SIFT algorithm to the statistical specificities of SAR images. Section II presents the outline of the classical SIFT algorithm and its behaviour on SAR images. Section III introduces a new gradient computation and a SIFT-like algorithm, both adapted to SAR images. Experimental validations and performances are presented in Section IV. Finally, section V investigates the possibilities offered by this new algorithm for multi-angles and multiresolutions contexts in SAR imaging. An application of SAR image registration and preliminary results for change detection are presented.

A conference proceedings version of this work has appeared in [20].

\section{Presentation of the SiFt Algorithm AND BEHAVIOUR ON SAR IMAGES}

In this section the original SIFT algorithm and some of its variants are introduced. We also present its limitations when applied to SAR images and some of its adaptations to cope with such images.

\section{A. Presentation of the original SIFT algorithm}

The SIFT algorithm has been introduced by Lowe in 2004 [1] for the matching of local features in natural images. The algorithm follows four steps, that we describe in the following paragraphs:

1) Keypoints detection: First keypoints are selected as local interest points and characterized by their localization $(x, y)$, scale $\sigma$ and orientation $\theta$ :

$$
P(x, y, \sigma, \theta) .
$$

A difference of Gaussian (DoG) [1] scale-space, as an approximation of the Laplacian of Gaussian (LoG) [21], is constructed with scales $\sigma_{l}=\sigma_{0} \cdot r^{l}$ and $l \in \llbracket 0 . . l_{\max }-1 \rrbracket$. Local extrema in the three dimensions $(x, y, \sigma)$ are then selected to obtain keypoints defined by their position and scale.

Candidates with low contrast or located on the edges are filtered by a criterion based on the Hessian matrix [1]. Another possibility is to use the multi-scale Harris corner detector, based on the Harris matrix [22].

Among other interest point detectors, we can cite HarrisLaplace [23] that localizes points in space as extrema of the multi-scale Harris function and in scale as maxima of the LoG. More accurate localization is achieved by Hessian-Laplace [24] by replacing the space selection with local maxima of the Hessian determinant. To achieve affine invariance, Harris-Affine [25] and Hessian-Affine [24] detectors refine the localization with an iterative adaptation based on the second-order derivative matrix. ASIFT [26] simulates different viewpoints to evaluate two camera axis orientation parameters.

In this paper we will compare the proposed approach for keypoints detection (see Section III-B1) to keypoints that are detected as local extrema (in $(x, y, \sigma)$ ) in the LoG scale-space. These points will be filtered by the multiscale Harris criterion to eliminate those lying on edges or low-contrasted. We will refer to this approach as the LoG method.

2) Orientation assignement: To determine the main orientations associated with keypoints, Lowe [1] suggests to compute a local histogram of gradient orientations, weighted by the gradient magnitudes. The histogram is computed on a scaledependent neighborhood. The main orientations are defined as bins superior to $80 \%$ of the maximum. In [27], the histogram is replaced by Haar wavelet responses in $x$ and $y$ directions and the sum of responses is computed within a sliding orientation window to estimate the principal orientation.

In this paper, following [28], we select the main modes of the local orientation histogram thanks to an a contrario approach. As in the original SIFT algorithm, different keypoints can be obtained with the same position and scale but with different orientations $\theta$.

3) Descriptors extraction: A SIFT descriptor is assigned to each keypoint $P(x, y, \sigma, \theta)$ to describe its local geometry. A square neighborhood is defined around each point with a size depending on $\sigma$ to obtain translation and scale invariance. It is then rotated by an angle $-\theta$ to ensure rotation invariance. This normalized neighborhood is divided into $4 \times 4$ square sectors, upon which histograms of the gradient orientations, weighted by the gradient magnitudes, are computed. For each keypoint, the SIFT descriptor is obtained by concatenating and normalizing these histograms.

Different adaptations of the SIFT descriptor have been proposed in the literature. PCA-SIFT [29] is obtained by applying PCA on normalized gradient neighborhood. GLOH [2] is computed on a log-polar grid and upon 17 sectors, the size of the resulting vector being reduced with PCA. SURF [27] is obtained by replacing gradient histograms by sums of Haar wavelet responses in vertical and horizontal directions.

Here we choose to use the SIFT descriptor with a log-polar grid [28] of 9 sectors (Fig. 11).

4) Keypoints matching: Keypoints of two different images are matched according to their respective descriptors. Different matching criteria exist in the literature but the most commonly used is the Nearest Neighbor Distance Ratio (NNDR) method [1]. First, euclidean distances are computed between one descriptor and the ones of the other image and the nearest neighbor is chosen. To filter false matches, distances to the second and first closest neighbor are compared. A threshold th is applied on the ratio of those respective distances. We will further call the first step as the Nearest Neighbor (NN) step and the second as the Distance Ratio (DR) step.

In [28], a probability of false alarm is computed for all possible matches using an a contrario method. This approach allows different matches for one keypoint and permits to recognize multiple occurences of one object.

For the sake of simplicity, the NNDR method will be used here. 


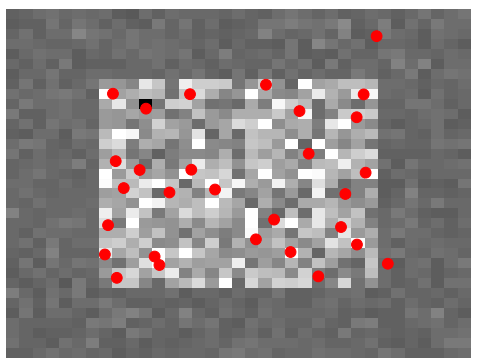

Fig. 1: Results of the LoG keypoints detection method applied on a rectangle corrupted by speckle noise and 1-look amplitude image (29 keypoints detected).

\section{B. Limitations of the SIFT algorithm on SAR images}

The SIFT algorithm does not perform well on SAR images. Many false keypoint detections as well as false matches occur. In particular, speckle noise leads to numerous false detections with the LoG method (Fig. 1). On optical images, noise is usually relatively weak and the keypoints filtering part of the algorithm (multi-scale Harris criterion [22]) suppresses most of the false detections thanks to its contrast dependency. However SAR images present a large dynamic range and the multiplicative noise leads to stronger gradient magnitude on homogeneous areas with high reflectivity (Figure 2(b)). False alarms on high contrast areas are thus not suppressed, as seen on the example of a rectangle corrupted by speckle noise (see Figure 1).

The orientations and descriptors are also not robust to multiplicative noise, since their computation relies on a classical gradient by difference.

\section{Previous adaptations of the SIFT algorithm for SAR images}

Modifications of the SIFT algorithm for SAR images have already been proposed in the literature. Some suggest to simplify the algorithm, by skipping the smallest scales for the keypoints detection [12] or by suppressing the orientation assignement [13], [17]. While such a procedure does decrease the number of false detections since many occur at those scales, the remaining keypoints are still not precisely located. Suppressing the orientations limits the capability of the algorithm to match images with different viewing conditions.

To improve the algorithm, some steps can be adapted. In [15], intensity values are thresholded to obtain spacially uniformly distributed keypoints and the size of the region descriptor is extended to increase matching performances. But this limits the distinctiveness of descriptors and prevents the application of the algorithm on images with strong changes. In [14], a new pyramid with progressive downsampling is used for keypoints detection and the SIFT descriptor is replaced by an improved version of Shape Context. While faster, this new algorithm has lower performances than the original SIFT.

Some works propose to reduce the influence of speckle by replacing the Gaussian scale space by an anisotropic one [30] or by computing multi-looks [19], [18]. But this last process decreases image resolution and causes loss of information.
Another solution is to denoise the images : curvelet transformation [10] or Infinite Symmetric Exponential Filter (ISEF) [11] can be used. Denoising is time consuming and can create artefacts that disturb the algorithm. While the performances of these algorithms are better than those of the original SIFT algorithm directly applied to SAR images, the number of correct matches is usually low (of the order of a few dozens).

Other studies suggest to improve performances by rejecting outliers. Lv [16] divides the images into four subregions and considers the spatial relationships of the matched keypoints in every subregion. This however implies that the images represent the same scenes with almost no overlaps and no rotation. For a registration application, image transformation is estimated in [17] based on best correspondences but a restrictive deformation is used. $\mathrm{Wu}$ [31] combines the SIFT algorithm and the cluster reward similarity measure to estimate iteratively an affine transformation. The process is time consuming and restricted to image registration.

The search space can be limited by performing a manual pre-registration [19]. False correspondences can be removed by knowing orbit informations, even if not precise [18], and a digital elevation model (DEM). But these kinds of informations are not always known and manual preregistration is time consuming and subject to interpretation errors.

To adapt the SIFT algorithm to SAR images, it is necessary to take into account the statistical specificities of SAR images. We suggest to develop first a new gradient computation thanks to which both the magnitude and the orientation are robust to speckle noise. Several steps of the algorithm can then be adapted to SAR images. A new keypoints detection method is introduced, as well as a new orientation assignement and a SAR adapted descriptor. The keypoints matching step is not modified, since it does not depend much on the type of images but rather on the quality of the descriptors. Section III introduces these new developments.

\section{PRoposed METHOD}

\section{A. Gradient computation for SAR images}

1) State of the art: Many works on edge detection have underlined the problem of using gradient by difference on SAR images. Indeed, variances of the gradient components depend on the underlying reflectivities [32]. Traditional approaches in edge detection consist in thresholding the gradient magnitude. For SAR images, this leads to higher false alarm rates in homogeneous areas of high reflectivity than in the ones of low reflectivity. The classical gradient by difference is thus not a constant false alarm rate operator. Statistical studies [32], [33], [34] have shown that the use of ratio is more suitable to multiplicative noise than the use of difference. Several edge detectors using ratio have been introduced in order to obtain a constant false alarm rate on SAR images:

- The Ratio of Average (ROA) [32], [33] consists in computing the ratio of local means on opposite sides of the studied pixel along one direction $i$ (Figure 3(a)): 


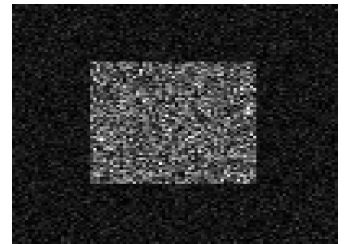

(a) Rectangle corrupted by speckle noise

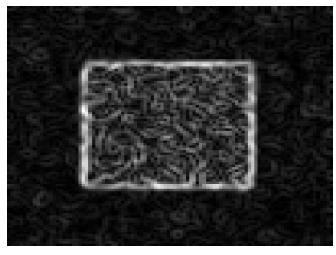

(b) Gradient by difference (Eq. $(8,9))$ and

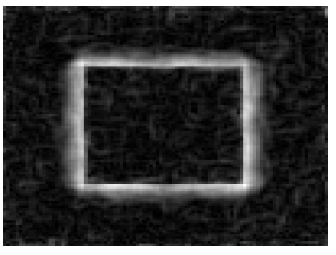

(c) Gradient by Ratio
Fig. 2: Example of a rectangle corrupted by speckle noise and its gradient magnitude for two gradient computation methods.

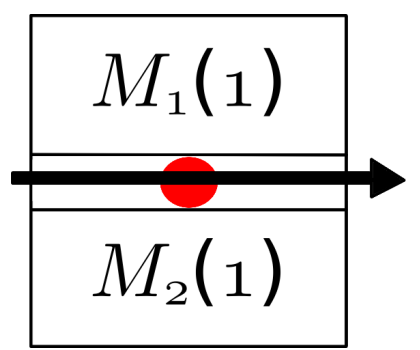

(a) Scheme of the ratio of local (b) means for the first direction.

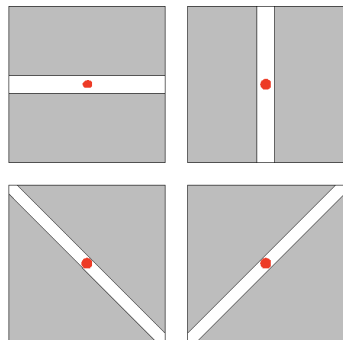

Four main directions, to compute respectively $T_{3}, T_{1}, T_{2}$ and $T_{4}$.
Fig. 3: Scheme of the ROA method [32], [33].

$$
R_{i}=\frac{M_{1}(i)}{M_{2}(i)} .
$$

The ratio $R_{i}$ is then normalized:

$$
T_{i}=\max \left(R_{i}, \frac{1}{R_{i}}\right) .
$$

These ratios are computed along the four main directions (Figure 3(b)). The gradient magnitude $D_{n}^{1}$ and orientation $D_{t}^{1}$ are defined as:

$$
\begin{aligned}
& D_{n}^{1}=\max _{i}\left(T_{i}\right) \\
& \left.D_{t}^{1}=\underset{i}{\operatorname{argmax}}\left(T_{i}\right)-1\right) \times \frac{\pi}{4} .
\end{aligned}
$$

Edges may then be obtained by thresholding the gradient magnitude $D_{n}^{1}$.

- The Ratio of Exponentially Weighted Averages (ROEWA) [35] is an improvement of the ROA for a multi-edge context, obtained by computing exponential weighted local means (Figure 4). For example, given a point $(a, b)$, the means are defined for the vertical direction as:

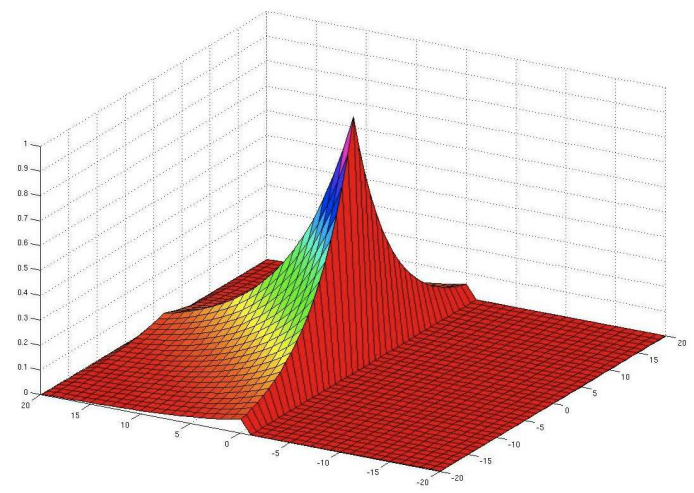

Fig. 4: Exponential filter for computation of weighted means.

$$
\begin{aligned}
& M_{1, \alpha}(1)=\int_{x=\mathrm{R}} \int_{y=\mathrm{R}^{+}} I(a+x, b+y) \times e^{-\frac{|x|+\alpha|y|}{\alpha}} \\
& M_{2, \alpha}(1)=\int_{x=\mathrm{R}} \int_{y=\mathrm{R}^{-}} I(a+x, b+y) \times e^{-\frac{|x|+\alpha|y|}{\alpha}}
\end{aligned}
$$

with $\alpha$ the exponential weight parameter.

As in the ROA, the ratio and its normalization for a direction $i$ are defined as:

$$
\begin{aligned}
R_{i, \alpha} & =\frac{M_{1, \alpha}(i)}{M_{2, \alpha}(i)} \\
T_{i, \alpha} & =\max \left(R_{i, \alpha}, \frac{1}{R_{i, \alpha}}\right) .
\end{aligned}
$$

These ratios $T_{i, \alpha}$ are computed along the horizontal ( $i=$ $1)$ and vertical $(i=3)$ directions. By analogy to the edge detectors on optical images that are based on gradients, the edge image is obtained by:

$$
D_{n, \alpha}^{2}=\sqrt{\left(T_{1, \alpha}\right)^{2}+\left(T_{3, \alpha}\right)^{2}} .
$$

The ROEWA is more precise in a multi-scale edge context and more robust to noise than the ROA, since the weighting parameter $\alpha$ allows an adaptive smoothing of the image.

Those operators have been designed for edge detection and provide a good estimate of the gradient magnitude. However they do not give a precise measure of the gradient orientation since only a few directions are considered. This could be improved by increasing the number of directions, but it would be time consuming.

Suri [13] proposes to define the vertical and horizontal gradient as respectively $T_{1, \alpha}$ and $T_{3, \alpha}$. By analogy to the gradient-based edge detector for optical images, the gradient magnitude and orientation are estimated as:

$$
\begin{aligned}
D_{n}^{3} & =\sqrt{\left(T_{1, \alpha}\right)^{2}+\left(T_{3, \alpha}\right)^{2}} \\
D_{t}^{3} & =\arctan \left(\frac{T_{3, \alpha}}{T_{1, \alpha}}\right) .
\end{aligned}
$$




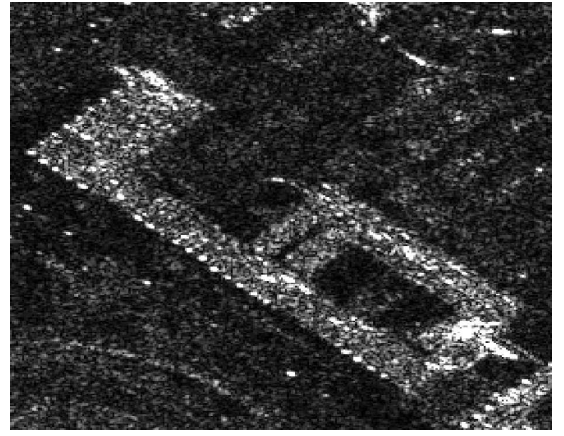

Fig. 5: Extract of Cosmo-SkyMed@image with $34^{\circ}$ of incidence angle and $1 \mathrm{~m}$ resolution.

This definition of orientation is highly questionable. Indeed, $T_{1, \alpha}$ and $T_{3, \alpha}$ always take positive values and thus $D_{t}^{3}$ can only take values between 0 and $\frac{\pi}{2}$. Moreover, the gradient computation on a vertical edge with reflectivities $m_{a}$ and $m_{b}$ $\left(m_{a}<m_{b}\right)$ yields:

$$
\begin{aligned}
T_{1, \alpha} & =\frac{m_{a}}{m_{b}} \\
T_{2, \alpha} & =1 \\
D_{t}^{3} & =\arctan \left(\frac{m_{b}}{m_{a}}\right) .
\end{aligned}
$$

Therefore the gradient orientation takes arbitrary values depending on the reflectivities of the areas, while it is expected to be equal to zero. The normalized ratios $T_{1, \alpha}$ and $T_{3, \alpha}$ should not be used directly to compute the gradient orientation.

2) Proposed approach: We propose here to define the horizontal and vertical gradient as:

$$
\begin{aligned}
G_{x, \alpha} & =\log \left(R_{1, \alpha}\right) \\
G_{y, \alpha} & =\log \left(R_{3, \alpha}\right)
\end{aligned}
$$

and to compute the gradient magnitude and orientation in the usual way as:

$$
\begin{aligned}
G_{n, \alpha} & =\sqrt{\left(G_{x, \alpha}\right)^{2}+\left(G_{y, \alpha}\right)^{2}} \\
G_{t, \alpha} & =\arctan \left(\frac{G_{y, \alpha}}{G_{x, \alpha}}\right)
\end{aligned}
$$

with $\alpha$ the parameter of the exponential weight used to compute the local means.

By using the logarithm, the problem mentioned above for the gradient orientation on a vertical edge is avoided, since the computation yields:

$$
\begin{aligned}
G_{x, \alpha} & =\log \left(m_{a}\right)-\log \left(m_{b}\right) \\
G_{y, \alpha} & =0 \\
G_{t, \alpha} & =0 .
\end{aligned}
$$

There is no normalization with the minimum (or maximum) between the ratio and its inverse, in order to obtain negative and positive gradient values. With this approach the whole possibilities of orientation values are taken into account. Also since the weighting parameter $\alpha$ allows to smooth the

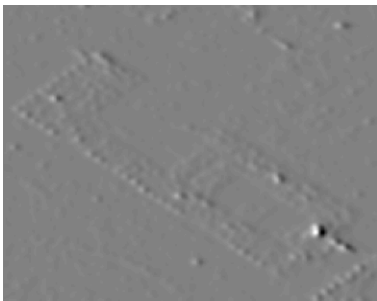

(a) Horizontal component

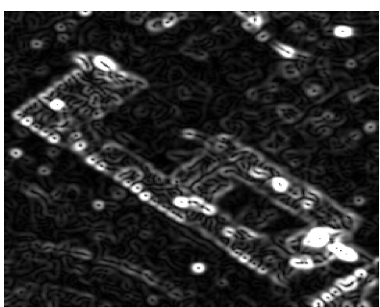

(c) Magnitude

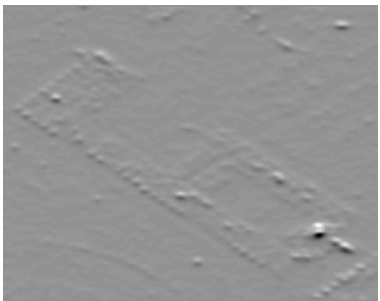

(b) Vertical component

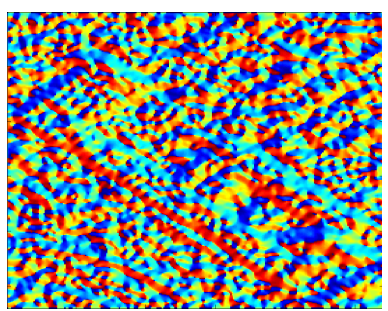

(d) Orientation
Fig. 6: Gradient by difference applied on the image of Fig. 5 with a Gaussian blur of $\sigma=2$.

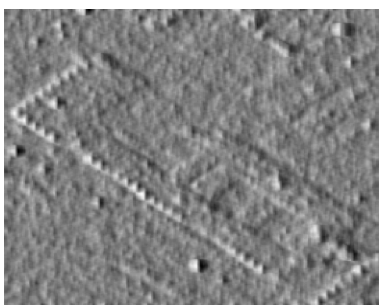

(a) Horizontal component

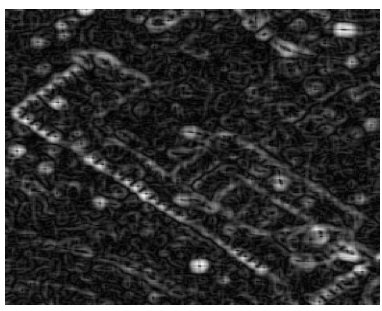

(c) Magnitude

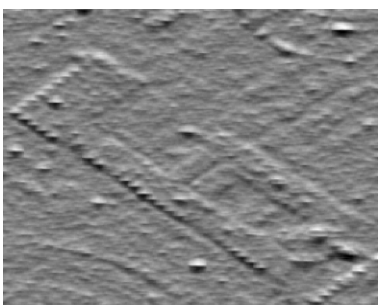

(b) Vertical component

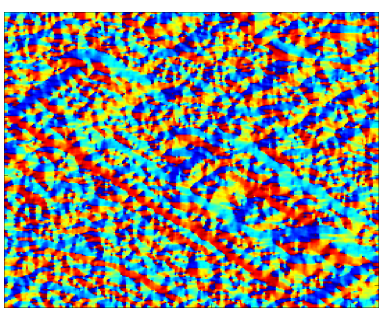

(d) Orientation
Fig. 7: New gradient computation (Gradient by Ratio) applied on the image of Fig. 5 with $\alpha=2$.

image at different scales, this gradient can be compared to the gradient by difference applied on an image with Gaussian blur. We call this new gradient computation method Gradient by Ratio (GR).

Figure 2 presents the gradient magnitude on a rectangle corrupted by speckle noise for the two gradient computation methods. The Gradient by Ratio method (Eq. $(8,9))$ does not produce more high values on high reflectivities areas than on the ones of low reflectivity, unlike the gradient by difference.

The gradient values of the image of Figure 5 are presented on Figure 6 for the gradient by difference and on Figure 7 for the GR. It appears that the gradient by difference 
presents a better reduction to speckle noise but gradient values (magnitude, and vertical and horizontal components) are higher on high reflectivy areas than on low reflectivity ones. For the GR method however, gradient responses are not higher on those areas.

This new gradient computation method will now help us to adapt the SIFT algorithm to SAR images.

\section{B. A SIFT-like Algorithm adapted to SAR images}

The outline of the new algorithm, that we have called $S A R-S I F T$, is presented in Figure 8. As mentioned before, the first three steps of the algorithm are adapted to SAR images and only the last one (matching) is left unchanged.

1) Keypoints detection: A first simple approach to detect keypoints on SAR images would be to apply the LoG method on the logarithm of the image. This allows to deal with an additive noise instead of a multiplicative one, and to suppress the false detections on the high reflectivity areas. Although appealing because of its simplicity, this approach is not robust enough to noise and does not improve much the performances of the original LoG approach (Fig. 1). The example of a rectangle corrupted by speckle noise on Figure 9(a) shows that keypoints are indeed found near corners, as expected, but badly located. There are fewer false detections than when applied directly on the amplitude image (Fig. 1) and they also happen equally on high and low reflectivity areas, but they are still numerous. By adapting the parameters on the multi-scale Harris criterion [22], the number of false detections can be decreased but so will the number of correct ones.

LoG and Hessian matrices do not seem convenient and easy to adapt to multiplicative noise since they rely on second derivatives. The multi-scale Harris function [23], in contrast, is based on the first derivative. From the new gradient computation adapted to SAR images that we have developed in Section III-A, we propose a new approach based on this detector.

The multi-scale Harris matrix and function are defined for optical images respectively as:

$$
\begin{aligned}
& C(x, y, \sigma)=\sigma^{2} \cdot \mathcal{G}_{\sqrt{2} \cdot \sigma} \star\left[\begin{array}{cc}
\left(\frac{\partial I_{\sigma}}{\partial x}\right)^{2} & \left(\frac{\partial I_{\sigma}}{\partial x}\right) \cdot\left(\frac{\partial I_{\sigma}}{\partial y}\right) \\
\left(\frac{\partial I_{\sigma}}{\partial x}\right) \cdot\left(\frac{\partial I_{\sigma}}{\partial y}\right) & \left(\frac{\partial I_{\sigma}}{\partial y}\right)^{2}
\end{array}\right] \\
& R(x, y, \sigma)=\operatorname{det}(C(x, y, \sigma))-t \cdot \operatorname{tr}(C(x, y, \sigma))
\end{aligned}
$$

with $\mathcal{G}_{\sqrt{2} \cdot \sigma}$ a Gaussian kernel with standard deviation $\sqrt{2} \cdot \sigma$, $\star$ the convolution operator, $I_{\sigma}$ the convolution of the original image by a gaussian kernel with standard deviation $\sigma$ and $t$ an arbitrary parameter. Observe that the weight $\sigma^{2}$ is needed here for full scale normalization [23]. In the LoG method, the multi-scale Harris criterion allows to suppress low-contrast and edge points by applying a threshold $d_{H}$ on $R(x, y, \sigma)$.

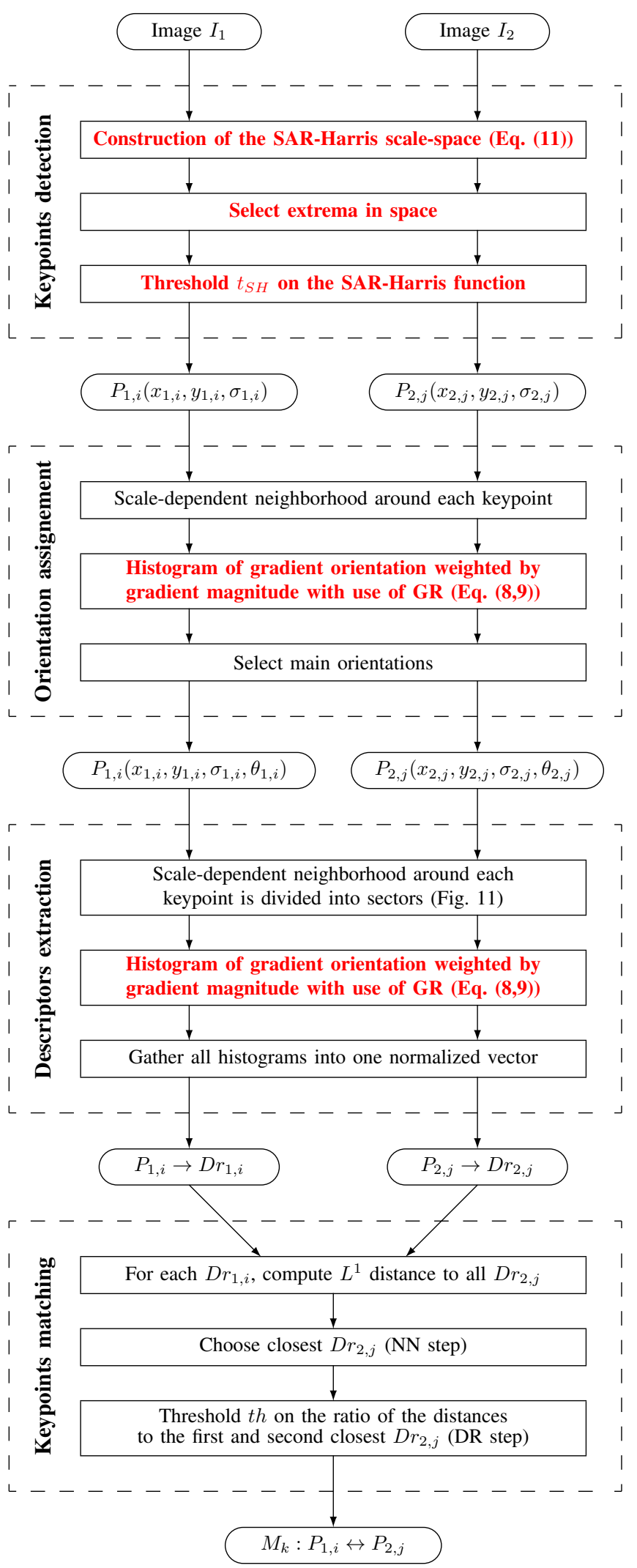

Fig. 8: Outline of SAR-SIFT algorithm. Contributions presented in this paper are in red. 


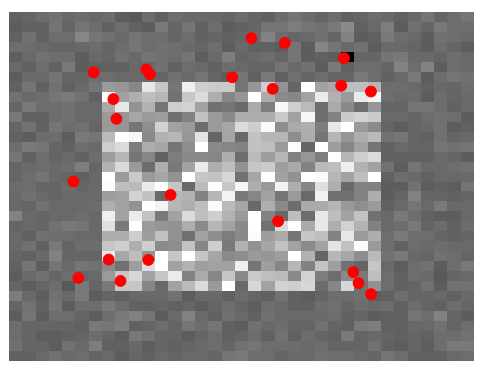

(a) LoG method applied on the logarithm image (22 keypoints)

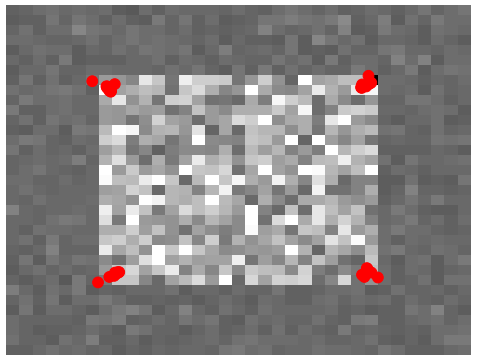

(b) SAR-Harris method (32 keypoints)

Fig. 9: Detection of keypoints on a rectangle corrupted by speckle noise with the LoG method applied on the logarithm of the image and the SAR-Harris method.

Considering this definition and the Gradient by Ratio, we propose the new SAR-Harris matrix and the multi-scale SARHarris function respectively as:

$$
\begin{aligned}
& C_{S H}(x, y, \alpha)=\mathcal{G}_{\sqrt{2} \cdot \alpha} \star\left[\begin{array}{cc}
\left(G_{x, \alpha}\right)^{2} & \left(G_{x, \alpha}\right) \cdot\left(G_{y, \alpha}\right) \\
\left(G_{x, \alpha}\right) \cdot\left(G_{y, \alpha}\right) & \left(G_{y, \alpha}\right)^{2}
\end{array}\right] \\
& R_{S H}(x, y, \alpha)=\operatorname{det}\left(C_{S H}(x, y, \alpha)\right)-d \cdot \operatorname{tr}\left(C_{S H}(x, y, \alpha)\right)
\end{aligned}
$$

with $d$ an arbitrary parameter, and where the derivatives $G_{x, \alpha}$ and $G_{y, \alpha}$ are computed using Eq. (8).

In this case, it can be shown that the multiplication by $\sigma^{2}$ is not needed anymore to ensure scale invariance.

For this keypoints detection method, we replace the LoG scale-space by a multi-scale representation of the original image, obtained by computing the multi-scale SAR-Harris function (Eq. (11)) at different scales $\alpha_{k}=\alpha_{0} \cdot c^{m}$ with $m \in \llbracket 0 . . m_{\max }-1 \rrbracket$. Local extrema in space are then selected at each level to be keypoints candidates. Subpixel positions of the keypoints are refined by performing a bilinear interpolation of the SAR-Harris criterion around the local extrema. A threshold $d_{S H}$ on the muli-scale SAR-Harris function allows to filter edge and low contrast points. We obtain keypoints characterized by their position $(x, y)$ and their scale $\alpha$.

This approach, called the SAR-Harris method, merges the two steps of the LoG method in order to avoid the use of second order derivatives. As it is easily verified, it also has the advantage of being independent of the image contrast.

We have noticed that this scale-space rarely reaches extrema in 3-dimensions. This fact was also observed on optical

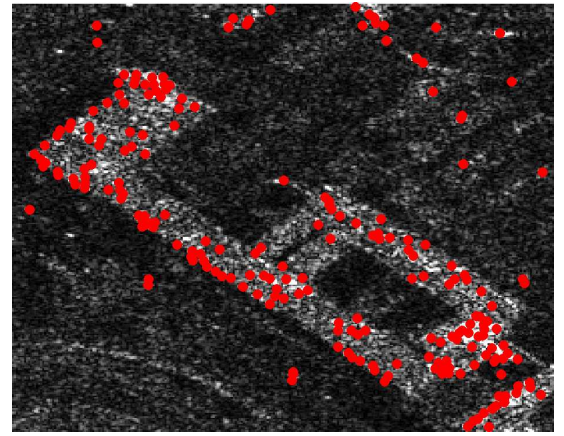

(a) LoG method applied on the amplitude image (435 keypoints)

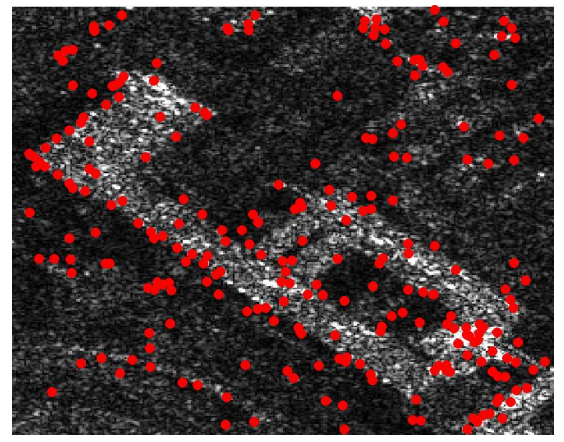

(b) LoG method applied on the logarithm of the image (435 keypoints)

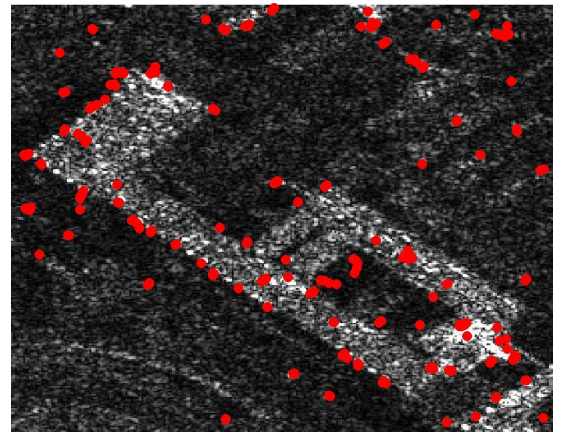

(c) SAR-Harris method (433 keypoints)

Fig. 10: Detection of keypoints on the image of Fig. 5 with the LoG method, applied on the amplitude image and the logarithm of the image, and the SAR-Harris method. The thresholds $d_{H}$ and $d_{S H}$ were adjusted to obtain the same number of keypoints regarding the keypoints detection method used.

images [23]. Only extrema in space are thus selected. Several detections can then occur at the same position but for different scales. However, some of them are suppressed by thresholding the multi-scale SAR-Harris function.

The example of a rectangle corrupted by speckle noise on Figure 9(b) shows the efficiency of this method: keypoints are only found on the corners, as expected, and there are no false detections.

Figure 10 presents an example of keypoints detection for different methods. As expected, we observe that keypoints 


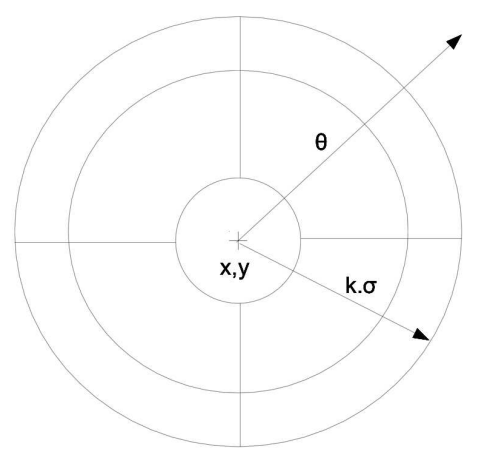

Fig. 11: Scheme of the circular descriptor. $k$ is a fixed parameter. Ratio of inner to outer circle radius is respectively 0.25 and 0.73 .

detected with the LoG method applied on the amplitude image are mainly detected on high reflectivity areas and a lot of false detections occur on high homogeneous areas. Concerning the keypoints detected with the LoG method applied on the logarithm of the image, a lot of false detections are also found on homogeneous areas but they happen both on high and low reflectivy areas. However, the keypoints detected with the SAR-Harris method are mostly located on corners and bright points, and the number of false detections on homogeneous areas is really low.

\section{2) Orientations Assignement and Descriptors Extraction:}

In the original SIFT algorithm, both the steps of orientation assignement and descriptor extraction rely on histograms of gradient orientation. These histograms are computed on a neighborhood of each keypoint and weighted by the gradient magnitude.

Here we propose to use the Gradient by Ratio (GR) method, introduced in Section III-A to compute those histograms. Instead of using a square neighborhood and $4 \times 4$ square sectors as in the original SIFT descriptor, we rely on a circular neighborhood (size of $6 \sigma$ ) and log-polar sectors as in [28], see Figure 11. The resulting descriptor is called Ratio Descriptor. Let us observe that the GR method to compute the gradient could straightforwardly be adapted to other spatial configurations of sectors, such as the one of the original SIFT.

In order to select principal orientations, we rely on an $a$ contrario approach [28]. Up to two orientations are selected at each points.

\section{EXPERIMENTAL VALIDATION OF THE SAR-SIFT ALGORITHM UNDER SPECKLE NOISE}

In this section, both the proposed keypoints and descriptors are compared to the original SIFT algorithm. We first investigate the stability and robustness of the keypoints detection methods by measuring their repeatability rate. Then performances of keypoints detection methods and descriptors are evaluated with the help of ROC curves. To only assess the ability of the algorithms to deal with speckle noise, the study is conducted on image pairs acquired under the same conditions.

\section{A. Test images and parameters}

For these experiments, we use 18 pairs of extracts of TerraSAR-X images with a subpixel registration, representing the city of Toulouse, France. All images have a size of $512 \times 512$ pixels and have been all acquired under the same viewing conditions $\left(34^{\circ}\right.$ incidence angle, $2 \mathrm{~m}$ resolution, SpotLight mode). A visual check showed that no temporal changes occur between the two images of each pair, so that only the noise realisation differs.

SIFT keypoints are detected with the LoG method (local extrema in LoG and threshold on the multi-scale Harris criterion). To construct the scale space, we choose the following parameters: $\sigma_{0}=0.63$ the first scale, $r=2^{1 / 3}$ the ratio between two scales and $l_{\max }=13$ the number of scales. For the multi-scale Harris criterion, the parameter $t$ is set to 0.04 . The threshold on this criterion $d_{H}$ is usually set to 2000 for 8-bits images, but will be adapted for each SAR image, since they have different dynamics.

For the SAR-Harris method, the chosen parameters are: $\beta_{0}=2$ the first scale, $c=2^{1 / 3}$ the ratio between two scales, $m_{\max }=8$ the number of scales and $d=0.04$ the arbitrary parameter of the SAR-Harris criterion. The threshold $d_{S H}$ has been set to 0.8 after an experimental study of the probability distribution of the SAR-Harris criterion computed on corners, borders and homogeneous areas.

For both the Ratio and SIFT descriptors, histograms are computed on 12 bins and the log-polar grid is used with $k=12$.

\section{B. Keypoints repeatability}

The repeatability criterion [36] gives a measure of the stability of keypoints detection, regarding the image changes. Given a pair of registered images, we look, for each keypoint of the first image, at the closest one extracted on the other image with the same method. Then for different thresholds $u$, the percentage of keypoints repeated on the other image at a distance lower than $u$ is observed.

The new SAR-Harris keypoints detection method presented in Section III-B1 is compared to the LoG method applied on either the amplitude image and the logarithm image. Results are shown on Figure 12. The thresholds $d_{H}$ have been adapted to obtain on average the same number of keypoints than with the SAR-Harris method. We obtain for the entire set a total of 25032 keypoints extracted with the original LoG method, 24729 keypoints when the logarithm of the image is used and 21253 keypoints with the SAR-Harris method. The keypoints density is thus the same for each detection method.

It can be observed that the SAR-Harris method performs better than the two LoG methods. For example at a localization error of $d=1.5$ pixels, more than $50 \%$ of the keypoints extracted by the SAR-Harris method are repeated. This rate is only $30 \%$ for the keypoints extracted with the two other methods. We also observe that the performances of the LoG 


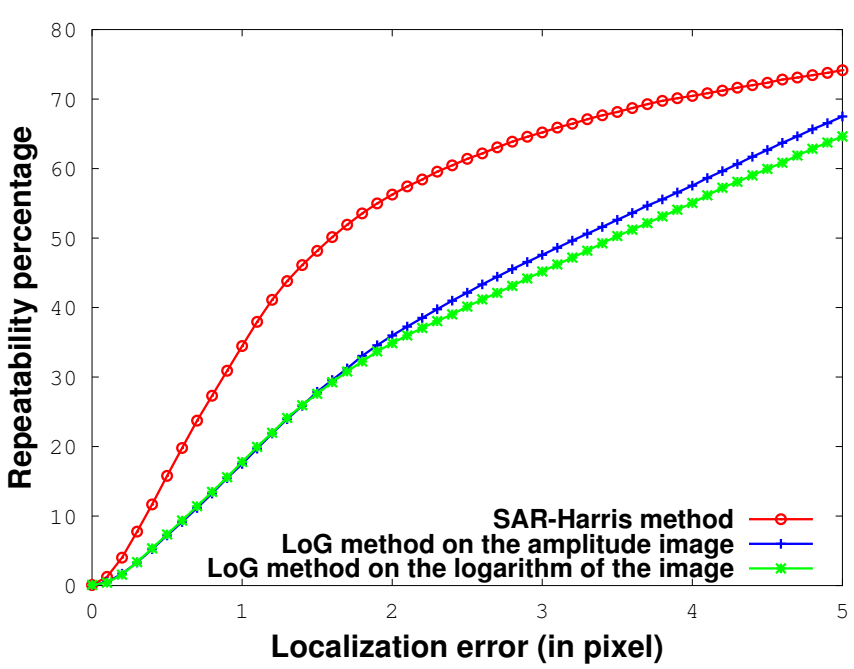

Fig. 12: Repeatability rate of keypoints, computed on 18 image pairs, with respect to the localisation error. Keypoints are extracted with three different methods: the LoG method applied on the amplitude image and on the logarithm of the image, and the SAR Harris method.

method do not present an improvement when applied on the logarithm image rather than on the amplitude one.

\section{Matching performances}

Global ROC curves are computed for different combinations of keypoints detection methods and types of descriptor. Keypoints are matched with the NNDR method (NN step then DR step, see Section II-A4).

Let $M_{k}\left(P_{1, i}, P_{2, j}\right)$ be a match between a point $P_{1, i}\left(x_{1, i}, y_{1, i}, \sigma_{1, i}, \theta_{1, i}\right)$ of an image $I_{1}$ and a point $P_{2, j}\left(x_{2, j}, y_{2, j}, \sigma_{2, j}, \theta_{2, j}\right)$ of an image $I_{2}$. Considering the deformation $T$ of the image $I_{1}$ in comparison to the image $I_{2}, M_{k}$ is defined as correct if:

$$
\left\|T\left(x_{1, i}, y_{1, i}\right)-\left(x_{2, j}, y_{2, j}\right)\right\|_{2}<t_{1} \cdot \min \left(\sigma_{1}, \sigma_{2}\right),
$$

where $t_{1}$ is set to 5 . Here the image pairs present a subpixel registration, so $T$ is the identity function.

The quantities $\# C M_{\text {all }}$ and $\# F M_{\text {all }}$ are defined respectively as the total number of correct and false matches for the entire set with the NN step. The number of correct and false matches, respectively $\# C M$ and $\# F M$, is evaluated for a certain value of the threshold th on the ratio of the distance to the closest and second closest match (DR step). To obtain Receiver Operating Characteristic (ROC) curves, the percentage of correctly matched keypoints $\frac{\# C M}{\# C M_{\text {all }}+\# F M_{\text {all }}}$ is ploted against the false alarm rate $\frac{\# F M C M_{\text {all }}+\# F M_{\text {all }}}{\# C M+\# F M}$ by varying th.

We compare here two keypoints detection methods, the LoG method on the amplitude image and the new SAR-Harris method, as well as two descriptors, the proposed Ratio and the usual SIFT descriptors. The results of the four considered

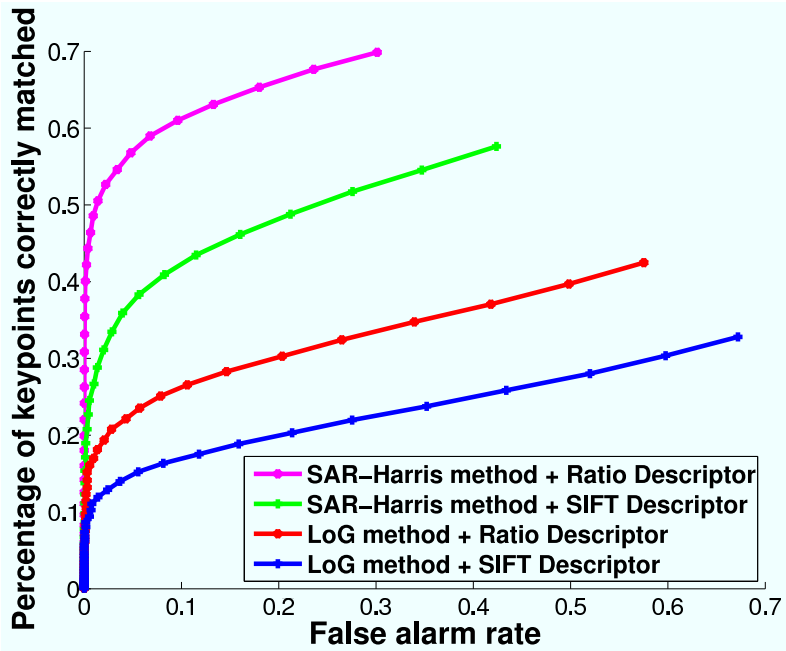

Fig. 13: Global ROC curves, computed on 18 image pairs, to evaluate the performance of the Ratio and SIFT descriptor, and the LoG and SAR-Harris methods.

situations are displayed on Figure 13. Since the images do not present any rotation, no orientations are assigned to the keypoints for this experiment, neither for the SIFT nor the SAR-SIFT approach. The $L^{1}$ and $L^{2}$ distances are tested to compute similarities between descriptors at the matching step. We have observed that results with the $L^{1}$ distance are always better than with the Euclidean distance. To simplify the reading, only matches with the $L^{1}$ distance are displayed.

We observe that the best performance is achieved by the combination of the SAR-Harris keypoint detection method and the Ratio Descriptor. Indeed, for a false alarm rate of $1 \%$, almost $50 \%$ of the possible correct matches are obtained, when for the other configurations this rate is less than $30 \%$. The $1 \%$ false alarm rate is the percentage of false matchs among the correspondences obtained with a certain value of $t h$, and not among all the possible matches. Also, using the SAR-Harris method with the SIFT descriptor already improves significantly the performances of the algorithm. In contrast, the use of the LoG method with the Ratio Descriptor offers a limited enhancement.

In summary, the SAR-Harris method is more stable and robust to noise than the LoG method. and the Ratio Descriptor outperforms the SIFT Descriptor. Combination of SAR-Harris and Ratio Descriptor are used in the SAR-SIFT algorithm (Fig. 8). This algorithm thus achieves better results than the original SIFT algorithm on SAR images.

\section{EXPERIMENTAL VALIDATION OF THE SAR-SIFT ALGORITHM IN MORE COMPLEX SITUATIONS AND APPLICATIONS}

After having validated experimentally the efficiency of the SAR-SIFT algorithm in dealing with strong SAR noise, we analyse its behaviour in more complex situations, and in particular with different acquisition modes. The use of the 
RANSAC algorithm is then suggested to increase the number of correct matches while keeping a low false alarm rate for some specific situations where a global deformation exists. Finally, two applications of the SAR-SIFT algorithm are considered, registration and change-detection.

\section{A. Behaviour of the SAR-SIFT with different image viewing conditions}

We focus now our study on image pairs with different resolutions and/or different incidence angles. In order to have a reference situation, we also consider image pairs having the same viewing conditions. We can expect some good results for the situations with only a difference in resolution. The situation is more complex when the incidence angle is changed, since the image geometry and the SAR signal are different. Therefore it can be expected that the number of associated keypoints will decrease in this case. This section investigates the variability of this number in different conditions.

The available sets of images are presented in Table I, with their characteristics (sensor, incidence angle, resolution, mode), number of images and size in pixels.

For the images with the same viewing conditions, matches were computed between every images of the sets $a, b$ and $c$. To obtain a situation with a varying resolution, a multilook has been computed in the azimuth direction for the images of the set $d$ to obtain a scale factor of two with respect to the images of the set $e$. For the case with difference in incidence angles, the sets $a$ and $b$ are used to study a case with a difference of incidence of $14^{\circ}$. Both are also compared with the set $c$, but in those cases there is also a difference in resolution. Five configurations are thus considered.

Ground truth deformation grids have been estimated manually ${ }^{1}$ between all considered images. The parameter $t_{1}$ is set to 5 , except for situations with incidence angle differences, where it is set to 7 . Indeed, due to slant-range distorsions, building sizes may vary in those cases and a more loose threshold should be considered. Here both SARSIFT and SIFT algorithms are performed with orientation assignement.

We first observe the ability of the algorithm to match corresponding keypoints using the NN step only (Section II-A4). We want to verify that the algorithm can obtain a sufficient number of correct matches, without considering the Distance Ratio step. Table II presents, for each configuration, the average number of keypoints extracted and correct matches per image with no thresholding.

Next, the DR threshold (allowing to reject false correspondances) is varied to produce ROC curves. Using the same notations as in Section IV-C, the percentage of good matches $\frac{\# C M}{\# C M_{\text {all }}}$ is plotted against the percentage of false

\footnotetext{
${ }^{1}$ Starting from a registration using the sensor parameters provided by space agencies, a fine manual registration is computed.
}

\begin{tabular}{|c|c|c|c|c|c|c|}
\hline \hline Set & Sensor & Angle & Resolution & Mode & Number & Size \\
\hline \hline$a$ & CSK & $48^{\circ}$ & $1 \mathrm{~m}$ & $\mathrm{D}$ & 4 & $2048 \times 2048$ \\
\hline$b$ & $\mathrm{CSK}$ & $34^{\circ}$ & $1 \mathrm{~m}$ & $\mathrm{D}$ & 2 & $2097 \times 1914$ \\
\hline$c$ & $\mathrm{CSK}$ & $43^{\circ}$ & $3 \mathrm{~m}$ & $\mathrm{D}$ & 6 & $646 \times 550$ \\
\hline$d$ & $\mathrm{TSX}$ & $34^{\circ}$ & $2 \mathrm{~m}$ & $\mathrm{~A}$ & 2 & $1500 \times 3000$ \\
\hline$e$ & $\mathrm{TSX}$ & $34^{\circ}$ & $1 \times 2 \mathrm{~m}$ & $\mathrm{~A}$ & 1 & $750 \times 1500$ \\
\hline
\end{tabular}

TABLE I: Available images, their characteristics, number for each set and size in pixels. TSX is the abbreviation for TerraSAR-X and CSK for Cosmo-SkyMed $\AA$. D is for Descending mode and A, for Ascending mode. All images were acquired on SpotLight mode.

\begin{tabular}{|c|c||c|c|}
\hline \hline $\begin{array}{c}\text { Scale } \\
\text { factor }\end{array}$ & $\begin{array}{c}\text { Difference of } \\
\text { incidence angle }\end{array}$ & $\begin{array}{c}\text { Number of } \\
\text { keypoints }\end{array}$ & $\begin{array}{c}\text { Number of } \\
\text { correct matches }\end{array}$ \\
\hline \hline- & - & 19393 & 11414 \\
\hline 2 & - & 36934 & 5199 \\
\hline 3 & $5^{\circ}$ & 50998 & 2251 \\
\hline 3 & $10^{\circ}$ & 50998 & 1529 \\
\hline- & $14^{\circ}$ & 49011 & 1144 \\
\hline
\end{tabular}

TABLE II: Average number, per image, of keypoints extracted and correct matches with the NN method for different configurations (scale factor and/or different incidence angles).

matches $\frac{\# F M}{\# C M+\# F M}$ for different values of $t h$, see Figure 14 .

As it can be expected, situations with either the same viewing conditions or difference in resolution present really good scores. The NN step allows a high percentage of keypoints to be correctly matched, respectively $59 \%$ and $14 \%$. Among these correct matches, roughly $80 \%$ and $45 \%$ respectively can be obtained with the DR step with only $1 \%$ of false alarms.

However, when considering an incidence angle difference, the scores are really low. Only a small percentage of keypoints (between 2 and 4\%) can be matched with the NN step. Since the number of extracted keypoints is large enough, a large amount of them are still correctly matched. But the DR part of the matching is not efficient enough to filter false correspondances and it is highly difficult to obtain a sufficient number of correct matches with a low false alarm rate. It is interesting to note that the influence of the resolution difference is very limited, unlike the incidence angle. Indeed the matching between the sets $a$ and $b$, with an incidence angle difference of $14^{\circ}$ and the same resolution, shows weaker scores than the matching between the sets $b$ and $c$, and $a$ and $c$, both with a scale factor of 3 and an incidence angle difference of respectively $9^{\circ}$ and $5^{\circ}$.

On images with the same viewing conditions or with only a resolution difference, image geometry barely varies and SARSIFT achieves a high number of correct keypoints matching (always more than a thousand). However, with different incidence angles, SAR signal varies and the corresponding distorsions are not taken into account in the invariances of SAR-SIFT. The larger the difference is, the greater the image variations are and the more difficult it is for SAR-SIFT to find corresponding keypoints. 


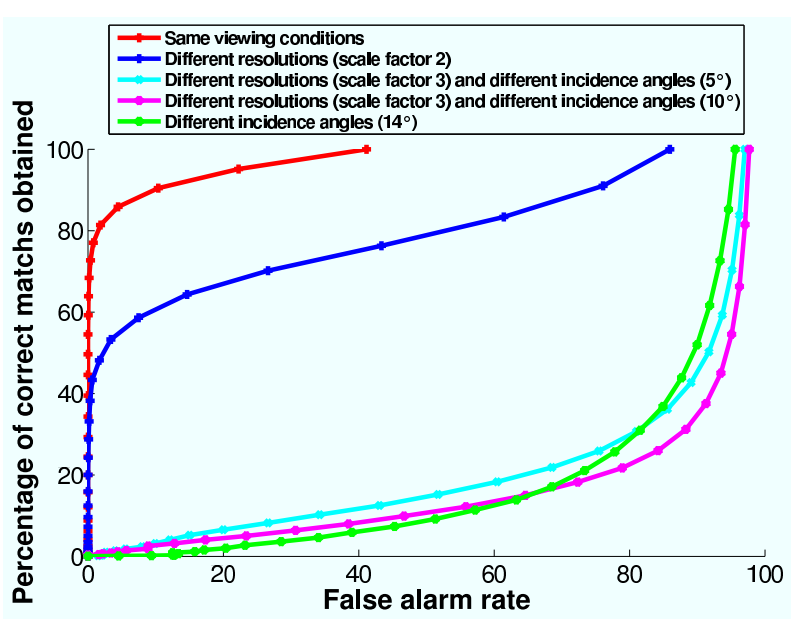

Fig. 14: Global ROC curves, computed with the images of Table I, to assess the number of SAR-SIFT matches with different acquisition conditions.

\section{$B$. Filtering of false matches to search a global deformation: AC-RANSAC}

1) Proposed approach: As seen in the previous paragraph, the filtering part of the keypoints matching (DR step) fails in situations with differences of incidence angles.

However, for registration purpose and as first approximation, satellite image pairs may be related by a global deformation. In multiview contexts and on roughly flat scenes, this deformation is often modeled by an affine transformation [37]. SAR images do present local displacements when acquired under different incidence angles. But the global relation between two SAR images can be described by this model, provided that they are acquired by the same sensor and in the same mode.

This a priori information can be helpful to suppress false correspondences in registration applications. We propose to use the RANSAC algorithm [38] in order to obtain a high number of correct matches with a low false alarm rate. This algorithm can be used to estimate a global deformation in presence of outliers (false correspondences) and thus to provide a set of coherent correspondences. We have chosen to use an a contrario version of RANSAC, called AC-RANSAC [39] that yields good results even with a very high percentage of outliers (up to $90 \%$ ). It also has the advantage to require only one parameter, the number of iterations $i_{\max }$.

2) Experimental results: AC-RANSAC has been applied on the correspondences of the images of the fifth situation of Table II, presenting the largest incidence angle difference. In order to obtain the highest number of correct matches, the DR filtering part is not applied. We call simple matches all the resulting correspondences using the NN step only. However, to speed up the process, a random selection of matches used to estimate the deformation is done among the correspondences with a ratio of distances lower than the threshold value of $t h=0.9$. We have chosen ${ }_{i \max }=10000$ as number of iterations and affine transformation as global deformation. Average number of false and true correspondences per

\begin{tabular}{|c||c|c|}
\hline \hline Studied situation & Simple matchs & AC-RANSAC \\
\hline \hline Correct matches & 2251 & 1979 \\
\hline False matches & 48747 & 104 \\
\hline
\end{tabular}

TABLE III: Number of correct and false matches before and after applying AC-RANSAC.

image are presented on Table III, before and after applying AC-RANSAC.

The use of AC-RANSAC allows to suppress almost all false matches while keeping $88 \%$ of the correct matches. The percentage of outliers goes from about $95 \%$ to $5 \%$. We can achieve a high number of correct matches with a reasonnable percentage of false alarms.

\section{Application of SAR-SIFT to Registration}

As explained in Section V-B, the deformation between two images acquired by the same sensor and in the same mode can be approximated by an affine transformation. This approximation however is only valid for points on the ground. AC-RANSAC has proven its efficiency to filter a significant number of false matches for registration purpose and can also be used to estimate the affine transformation between two images. We propose here a registration application of the SAR-SIFT algorithm in situations where the incidence angle is varying.

1) Proposed approach: To register such images, we need to estimate the coefficients of the following polynomial transformation:

$$
\begin{aligned}
& x_{2}=a_{1}+a_{2} \cdot x_{1}+a_{3} \cdot y_{1} \\
& y_{2}=b_{1}+b_{2} \cdot x_{1}+b_{3} \cdot y_{1}
\end{aligned}
$$

A least-square estimation of the coefficients can be done using the keypoint matchings between two images. However, as seen in Section V-A, the algorithm presents low efficiency in these conditions and we risk to face a significant number of outliers. As presented in Section V-B, the AC-RANSAC algorithm can help to model the deformation in presence of outliers and is efficient to filter false correspondences.

We propose to apply this algorithm on the matches obtained by the SAR-Harris algorithm between two SAR images in order to estimate the parameters of Eq. (13). The same process and parameters values presented in Section $\mathrm{V}-\mathrm{B}$ are used. This registration method will be further called SAR-SIFT + AC-RANSAC.

2) Evaluation: Table IV describes the pair of images used to assess the precision of the registration. They have the same resolution but a difference of incidence angle of $14^{\circ}$. Since there is no available ground truth, we have manually extracted 30 Ground Control Points (GCP) on the pair of images. These are then used to realize a manual registration as well as a measure of the registration accuracy.

Ten of the GCP are selected randomly to evaluate the parameters of Eq. (13) and obtain a manual registration. The twenty other points are used to measure the accuracy 


\begin{tabular}{|c|c|c|c|c|}
\hline \hline Sensor & Angle & Resolution & Mode & Size \\
\hline \hline Cosmo-Skymed $\mathbb{R}$ & $48^{\circ}$ & $1 \mathrm{~m}$ & Descendant & $2048 \times 2048$ \\
\hline Cosmo-Skymed $\mathbb{R}$ & $34^{\circ}$ & $1 \mathrm{~m}$ & Descendant & $2048 \times 2048$ \\
\hline
\end{tabular}

TABLE IV: Pair of images used to evaluate the precision of the registration performed by SAR-Harris + AC-RANSAC. Images are acquired on the area of Toulouse, France.

\begin{tabular}{|c||c|c|}
\hline $\begin{array}{c}\text { Registration } \\
\text { method }\end{array}$ & $\begin{array}{c}\text { Manual } \\
\text { registration }\end{array}$ & $\begin{array}{c}\text { SAR-SIFT } \\
\text { + AC-RANSAC }\end{array}$ \\
\hline \hline RMSE (in pixel) & 2.46 & 2.03 \\
\hline
\end{tabular}

TABLE V: Accuracy of the two registration methods for the images of Table IV.

of the two registration methods, the one using SAR-SIFT + AC-RANSAC and the previous manual one, by computing root mean square errors (RMSE). This process is repeated 10000 times and averaged RMSE are presented in Table V. Superposition of the two registered image by the SAR-SIFT + AC-RANSAC method is presented Figure 15.

The automatic method, SAR-SIFT + AC-RANSAC, presents a good registration accuracy, comparable to the manual method. Considering that extracting GCP is time consuming and subject to errors, this new method is an interesting way to automatically register SAR images with different types of acquisition.

\section{Change detection application: preliminary results}

Another possible application of SAR-SIFT would be change detection. Such applications are often subject to misregistration errors and the use of feature-based approaches allows to avoid a pre-registration step.

As a preliminary result, we match an image pair presenting some changes (Figure 16). One image has been acquired in

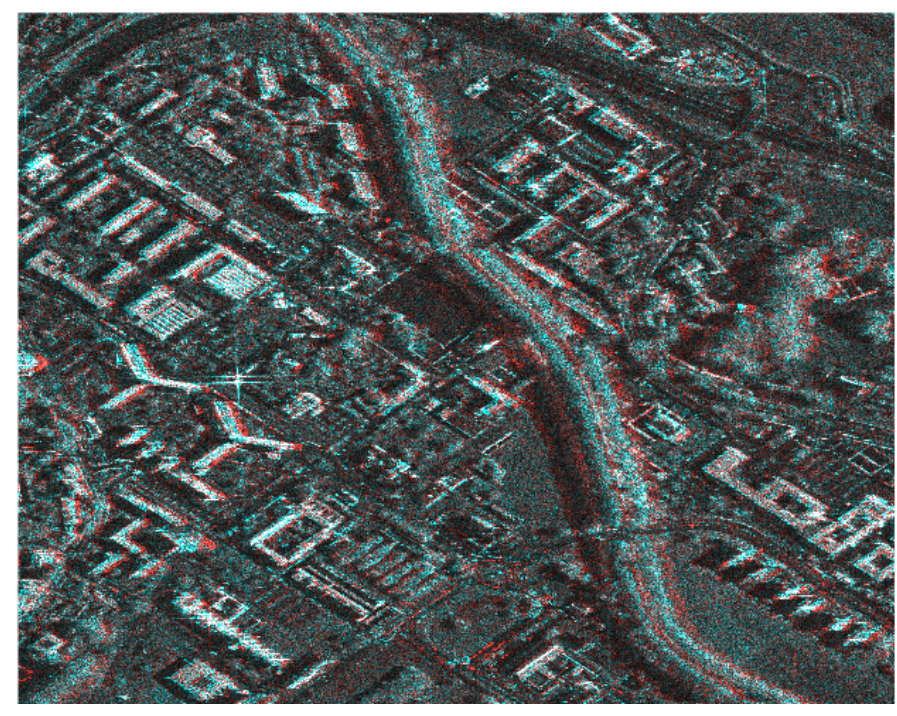

Fig. 15: Superposition of the two registered images (angular difference of $14^{\circ}$ ) of Table IV with the SAR-SIFT + ACRANSAC method. The master image is in red and the slave one in blue.

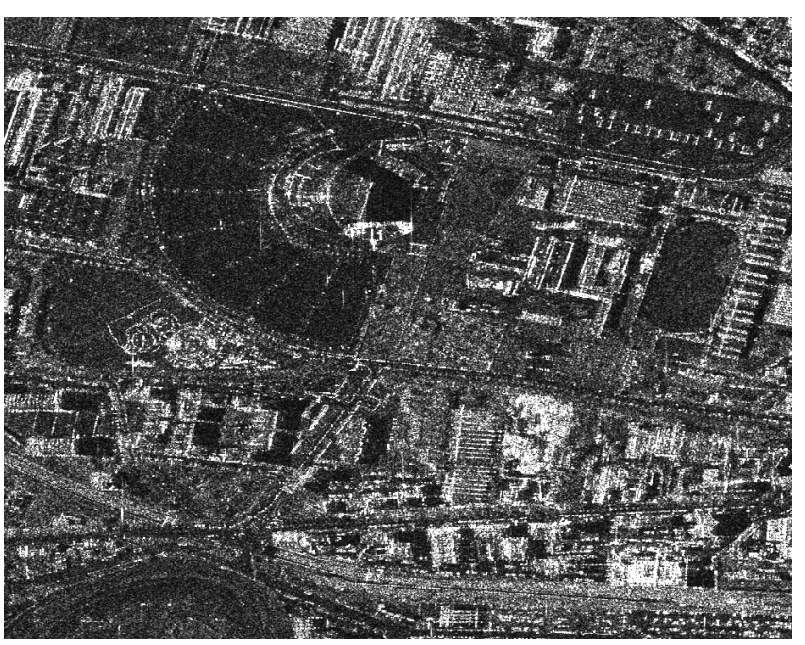

(a) Image from 2007

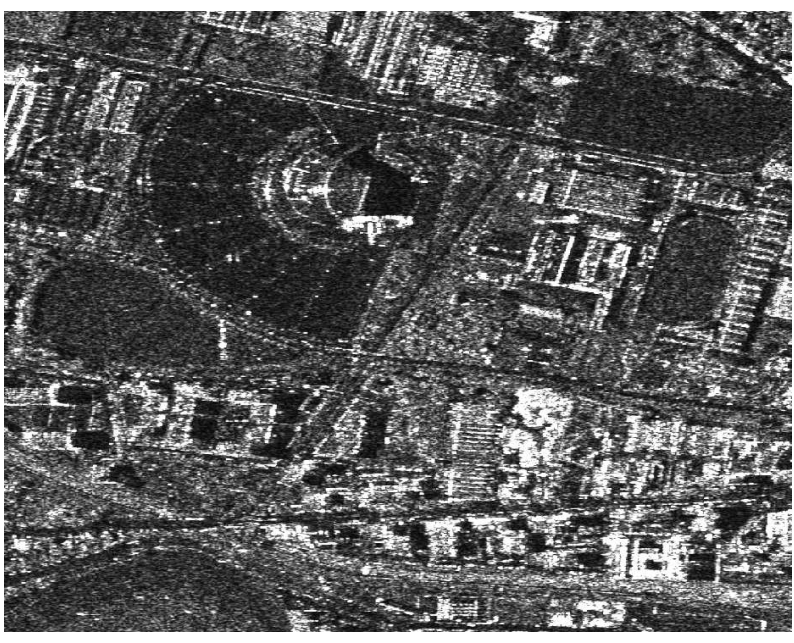

(b) Image from 2008

Fig. 16: Extracts of TerraSAR-X images with $34^{\circ}$ of incidence angle and $2 \mathrm{~m}$ resolution, from the area of Toulouse, France.

2007 and the other in 2008, see Figure 16. The obtained correspondances are then filtered with the AC-RANSAC algorithm. In area with changes, we suppose that keypoints are detected but not matched. In order to test this hypothesis the following experiment is conducted: Every keypoint at a distance lower than 40 pixels of a match is thus disgarded. Remaining keypoints from the 2007 image are displayed on Figure 17.

We observe that remaining points are present on the two main sites with changes, and that a few errors occur. Smaller changes are not detected, but the used algorithm is very simple and the tolerant threshold of 40 pixels is high.

From this preliminary experiment, we think that the SARSIFT algorithm can be considered for change detection applications. A more sophisticated algorithm should be developped, using keypoints density for example.

\section{CONCLUSiON}

This article presents a new SIFT-like descriptor adapted to SAR images. It relies on a new gradient computation adapted 


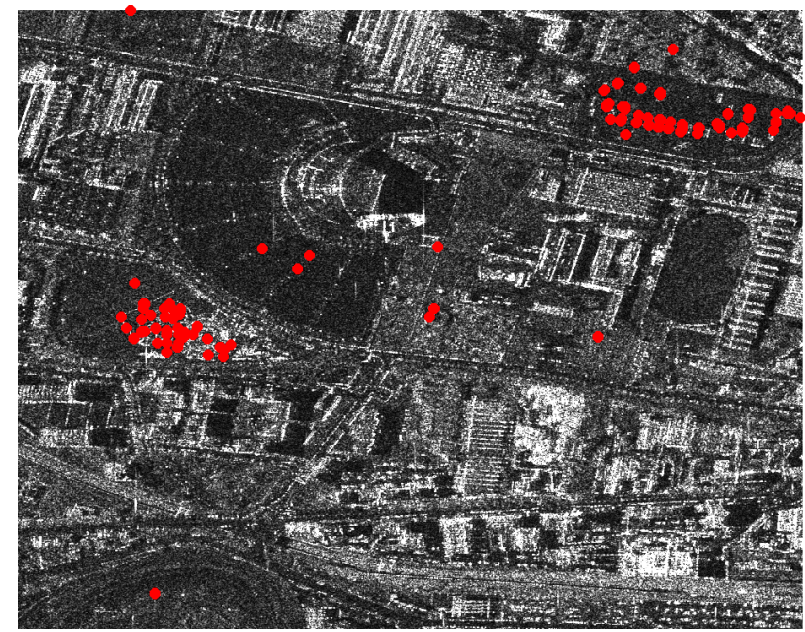

Fig. 17: The 2007 image is displayed with all keypoints that are at a distance of at least 40 pixels from a match between the 2007 and 2008 images.

to SAR images and robust to speckle noise. This new gradient computation method, Gradient by Ratio (GR), is then used to improve steps of the SIFT algorithm. A new keypoint detection method based on multi-scale Harris detector offers stable keypoints. Robust gradient orientations provided by GR enables to obtain a more efficient descriptor for SAR images than the original SIFT one.

By applying an a contrario RANSAC, a consistent number of correct matches can be achieved, allowing the use of this new SIFT-like algorithm for diverse applications. In this article, an efficient registration application of SAR images is presented for difficult situations, such as incidence angle changes. Other applications like change detection or object matching will be the subject of further work.

\section{ACKNOWLEDGEMENT}

The authors would like to thank the CNES and the ASI space agencies for the provided Cosmo-Skymed $\mathrm{R}$ images and the German Aerospace Center for the provided extracts of the TerraSAR-X images (RDLR 2007-2008. They also would like to thank Jean-Marie Nicolas for his help on registration with sensor parameters and Baptiste Mazin for the provided source codes of the SIFT algorithm.

\section{REFERENCES}

[1] D. G. Lowe, "Distinctive image features from scale-invariant keypoints," Int. J. Comput. Vision, vol. 60, pp. 91-110, 2004.

[2] K. Mikolajczyk and C. Schmid, "A performance evaluation of local descriptors," Pattern Analysis and Machine Intelligence, IEEE Transactions on, vol. 27, no. 10, pp. 1615-1630, oct. 2005.

[3] Qiaoliang Li, Guoyou Wang, Jianguo Liu, and Shaobo Chen, "Robust scale-invariant feature matching for remote sensing image registration," Geoscience and Remote Sensing Letters, IEEE, vol. 6, no. 2, pp. 287291, April.

[4] Chunlei Huo, Chunhong Pan, Leigang Huo, and Zhixin Zhou, "Multilevel SIFT matching for large-size VHR image registration," Geoscience and Remote Sensing Letters, IEEE, vol. 9, no. 2, pp. 171-175, 2012.

[5] A. Sedaghat, M. Mokhtarzade, and H. Ebadi, "Uniform robust scaleinvariant feature matching for optical remote sensing images," Geoscience and Remote Sensing, IEEE Transactions on, vol. 49, no. 11, pp. $4516-4527,2011$.
[6] Yi Yang and S. Newsam, "Geographic image retrieval using local invariant features," Geoscience and Remote Sensing, IEEE Transactions on, vol. 51, no. 2, pp. 818-832, 2013.

[7] V. Risojevic and Z. Babic, "Fusion of global and local descriptors for remote sensing image classification," Geoscience and Remote Sensing Letters, IEEE, vol. 10, no. 4, pp. 836-840, 2013.

[8] B. Sirmacek and C. Unsalan, "Urban-area and building detection using SIFT keypoints and graph theory," Geoscience and Remote Sensing, IEEE Transactions on, vol. 47, no. 4, pp. 1156-1167, 2009.

[9] Chao Tao, Yihua Tan, Huajie Cai, and Jinwen Tian, "Airport detection from large IKONOS images using clustered SIFT keypoints and region information," Geoscience and Remote Sensing Letters, IEEE, vol. 8, no. 1, pp. 128-132, 2011.

[10] J.Z. Liu and X.C. Yu, "Research on SAR image matching technology based on SIFT," in ISPRS08, 2008, p. B1: $403 \mathrm{ff}$.

[11] Sahil Suri, Peter Schwind, Peter Reinartz, and Johannes Uhl, "Combining mutual information and scale invariant feature transform for fast and robust multisensor SAR image registration," in 75th Annual ASPRS Conference, 2009.

[12] P. Schwind, S. Suri, P. Reinartz, and A. Siebert, "Applicability of the SIFT operator to geometric SAR image registration," Int. J. Remote Sens., vol. 31, no. 8, pp. 1959-1980, Mar. 2010.

[13] Sahil Suri, Peter Schwind, Johannes Uhl, and Peter Reinartz, "Modifications in the SIFT operator for effective SAR image matching," International Journal of Image and Data Fusion, vol. 1, no. 3, pp. 243256, 2010.

[14] J. Lu, B. Wang, H. M. Gao, and Z.Q. Zhou, "SAR images matching based on local shape descriptors," in Radar Conference, 2009 IET International, 2009, pp. 1-4.

[15] Lining Liu, Yunhong Wang, and Yiding Wang, "SIFT based automatic tie-point extraction for multitemporal SAR images," in Education Technology and Training, 2008. and 2008 International Workshop on Geoscience and Remote Sensing. ETT and GRS 2008. International Workshop on, 2008, vol. 1, pp. 499-503.

[16] Wentao Lv, Wenxian Yu, Junfeng Wang, and Kaizhi Wang, "SAR image matching based on SIFT keypoints and multi-subregions information," in Synthetic Aperture Radar (APSAR), 2011 3rd International Asia-Pacific Conference on, 2011, pp. 1-4.

[17] Bin Fan, Chunlei Huo, Chunhong Pan, and Qingqun Kong, "Registration of optical and SAR satellite images by exploring the spatial relationship of the improved SIFT," Geoscience and Remote Sensing Letters, IEEE, vol. 10, no. 4, pp. $657-661$, july 2013.

[18] B. Wessel, M. Huber, and A. Roth, "Registration of near real-time SAR images by image-to-image matching," in PIA07, 2007, p. 179.

[19] Yu Xiaoping, Liu Tong, Li Pingxiang, and Huang Guoman, "The application of improved SIFT algorithm in high resolution SAR image matching in mountain areas," in Image and Data Fusion (ISIDF), 2011 International Symposium on, 2011, pp. 1-4.

[20] F. Dellinger, J. Delon, Y. Gousseau, J. Michel, and F. Tupin, "SAR-SIFT: A SIFT-like algorithm for applications on SAR images," in Geoscience and Remote Sensing Symposium (IGARSS), 2012 IEEE International, 2012, pp. 3478-3481.

[21] Tony Lindeberg, "Feature detection with automatic scale selection," International Journal of Computer Vision, vol. 30, pp. 79-116, 1998.

[22] Yves Dufournaud, Cordelia Schmid, and Radu P. Horaud, "Matching images with different resolutions," in Proceedings of the IEEE Conference on Computer Vision and Pattern Recognition, Hilton Head Island, South Carolina, USA. 2000, pp. 612-618, IEEE Computer Society Press.

[23] K. Mikolajczyk and C. Schmid, "Indexing based on scale invariant interest points," in Computer Vision, 2001. ICCV 2001. Proceedings. Eighth IEEE International Conference on, 2001, vol. 1, pp. 525-531 vol.1.

[24] Krystian Mikolajczyk and Cordelia Schmid, "Scale and affine invariant interest point detectors," International Journal of Computer Vision, vol. 60, no. 1, pp. 63-86, 2004.

[25] K. Mikolajczyk and C. Schmid, "An affine invariant interest point detector," in Proceedings of the 7th European Conference on Computer Vision-Part I, London, UK, UK, 2002, ECCV '02, pp. 128-142, Springer-Verlag.

[26] Jean-Michel Morel and Guoshen Yu, "ASIFT: A new framework for fully affine invariant image comparison," SIAM J. Img. Sci., vol. 2, no. 2, pp. 438-469, Apr. 2009.

[27] Herbert Bay, Tinne Tuytelaars, and Luc Van Gool, "SURF: Speeded up robust features," in In ECCV, 2006, pp. 404-417.

[28] J. Rabin, J. Delon, and Y. Gousseau, "A statistical approach to the matching of local features," SIAM J. Img. Sci., vol. 2, pp. 931-958, September 2009. 
[29] Yan Ke and R. Sukthankar, "PCA-SIFT: a more distinctive representation for local image descriptors," in Computer Vision and Pattern Recognition, 2004. CVPR 2004. Proceedings of the 2004 IEEE Computer Society Conference on, 2004, vol. 2, pp. II-506-II-513 Vol.2.

[30] Shanhu Wang, Hongjian You, and Kun Fu, "BFSIFT: A novel method to find feature matches for SAR image registration," Geoscience and Remote Sensing Letters, IEEE, vol. 9, no. 4, pp. 649 -653, july 2012.

[31] Yingdan $\mathrm{Wu}$ and Ming Yang, "A multi-sensor remote sensing image matching method based on SIFT operator and CRA similarity measure," in Intelligence Science and Information Engineering (ISIE), 2011 International Conference on, 2011, pp. 115-118.

[32] R. Touzi, A. Lopes, and P. Bousquet, "A statistical and geometrical edge detector for SAR images," Geoscience and Remote Sensing, IEEE Transactions on, vol. 26, no. 6, pp. 764-773, nov 1988.

[33] A.C. Bovik, "On detecting edges in speckle imagery," Acoustics, Speech and Signal Processing, IEEE Transactions on, vol. 36, no. 10, pp. 1618 -1627 , oct 1988.

[34] C.J. Oliver, D. Blacknell, and R.G. White, "Optimum edge detection in SAR," Radar, Sonar and Navigation, IEE Proceedings -, vol. 143, no. 1, pp. $31-40$, feb 1996.

[35] R. Fjortoft, A. Lopes, P. Marthon, and E. Cubero-Castan, "An optimal multiedge detector for SAR image segmentation," Geoscience and Remote Sensing, IEEE Transactions on, vol. 36, no. 3, pp. 793-802, may 1998.

[36] Cordelia Schmid, Roger Mohr, and Christian Bauckhage, "Evaluation of interest point detectors," Int. J. Comput. Vision, vol. 37, no. 2, pp. 151-172, June 2000.

[37] Barbara Zitov and Jan Flusser, "Image registration methods: a survey," Image and Vision Computing, vol. 21, pp. 977-1000, 2003.

[38] Martin A. Fischler and Robert C. Bolles, "Random sample consensus: a paradigm for model fitting with applications to image analysis and automated cartography," Commun. ACM, vol. 24, no. 6, pp. 381-395, June 1981.

[39] Julien Rabin, Julie Delon, Yann Gousseau, and Lionel Moisan, "MACRANSAC: a robust algorithm for the recognition of multiple objects," in Proceedings of 3DPTV 2010, Paris, France, 2010, p. 051. 